\title{
Improving spatio-temporal focusing and source reconstruction through deconvolution
}

\author{
Brian E. Anderson ${ }^{(a)}$ \\ Geophysics Group EES-17, MS D443 \\ Los Alamos National Laboratory \\ Los Alamos, New Mexico 87545 \\ Johannes Douma \\ Center for Wave Phenomena \\ Colorado School of Mines \\ 1500 Illinois Street \\ Golden, Colorado 80401, USA
}

\author{
T.J. Ulrich \\ Geophysics Group EES-17, MS D443 \\ Los Alamos National Laboratory \\ Los Alamos, New Mexico 87545
}

Roel Snieder

Center for Wave Phenomena

Colorado School of Mines

1500 Illinois Street

Page 1 
Golden, Colorado 80401, USA

${ }^{(a)}$ bea@lanl.gov

Page 2 
Anderson, Wave Motion

${ }_{22}$ abstract

In this study, a technique is demonstrated to improve the ability of time reversal to both spatially and temporally focus, or compress, elastic wave energy, or to improve the quality of the reconstruction of the source signal. This method utilizes the deconvolution, or inverse filter, in single channel time reversal experiments in solids. Special attention is given to the necessary procedure for improving source signal reconstruction in real experimental conditions. It is also demonstrated theoretically and numerically that good temporal focusing implies that the radius in the spherically symmetric part of the spatial focus is small.

Keywords: time reversal; signal processing; communications 
Anderson, Wave Motion

31

\section{Introduction}

Time Reversal (TR) in acoustics has been the focus of much research [1, 2, 3, 4]. This has led to many applications in a wide variety of fields, such as medicine, communications, nondestructive evaluation (NDE), seismology, etc. In many of these applications, the ability to use TR depends upon its ability to precisely compress the measured, scattered waveforms to a point in both time and space, ideally a delta function, $\delta(t)$, which is commonly referred to as TR focusing. The desire to enhance the TR process to focus wave energy has led researchers to develop a variety of ways to accomplish this. Some techniques use arrays of input transducers, measure the wave field with an array near the desired focal spot, and then optimize the spatial and temporal focusing $[5,6,7,8,9,12,13,14,15]$. Other methods use an array of input transducers and optimize the temporal focusing at an output transducer $[16,17,18,19,20]$. Some of these techniques require, or at least benefit greatly from, large arrays, while others may enhance temporal focusing at the expense of spatial localization.

Another aspect of the focal signal that is of interest for certain applications, i.e., seismology (source mechanics identification), communications, NDE, or anywhere else where the focused signal fidelity is important, is the quality of the reconstruction of the source signal. As TR, in principle, recreates the source signal (in reverse, of course) interest has grown in this aspect of source recreation. In experimental TR studies, however, there is an inseparable transducer effect in this process. For this reason it would be desirable to find a method capable of removing this effect such that source reconstruction is enhanced.

We explore a simple approach and use deconvolution, a primitive, though robust, version of the inverse filter (IF) [5, 9], to provide an input signal to be used in the TR process. We then document what additional processing is necessary to either create an approximate delta function $\delta(t)$ (limited by available bandwidth) or a reconstruction of an arbitrary source function, $S(t)$, as an output at the focal point, and show laboratory ultrasound experiments 
where the temporal focusing obtained is superior to that obtained by using reciprocal time reversal. We also show the reconstruction of the source function, or a delta function as desired, is possible through the prescribed procedure. By scanning the sample near the focal point, we find that this improved temporal focusing is accompanied by a better spatial focusing. We then show theoretically that improved temporal focusing implies enhanced spatial focusing for the spherical average of the focus. The method described in this paper enhances both spatial and temporal focusing for a single channel and can be expanded to any number of channels. In contrast to many previous applications of the inverse filter [5, 6, 9], we also explore the effectiveness of using deconvolution for small time reversal mirrors (TRM), e.g., single channel[10, 11], in bounded media, with limited available bandwidth, and for the purpose of source reconstruction.

\section{Deconvolution as an inverse filter}

The time reversal process, a matched field process, is often used in an attempt to produce an impulsive focus, $\delta(t)$, of energy. This process utilizes a recorded impulse response, or Green function $G_{A B}$ between two points $A$ and $B$, a simple reversal in time of that signal and broadcast of it back into the same medium, from $A$ to $B$ or vice versa, to focus energy at the appropriate point in space $(A$ or $B)$; or

$$
\int_{-\infty}^{\infty} G_{A B}(\tau) G_{A B}(\tau-t) d \tau=\delta(t)
$$

where reciprocity has been used to replace the Green function $G_{B A}$ with $G_{A B}$. Equation (1) states that the autocorrelation of $G_{A B}(t)$ is ideally equal to a delta function. In practice, actual TR experiments cannot recreate a true delta function focus as one or more conditions necessary to satisfy Eq. (1) are typically violated. For example, due to either time, physical 
memory, or attenuation constraints, it is not possible to record for an infinitely long period of time. Also, the Green functions here are assumed to contain a flat, infinite bandwidth, which is routinely not the case in experiments particularly when using narrowband response transducers such as piezoelectric types. For this reason we, as others before us, asked the question: what would be required to most closely approach an impulse-like TR focus given typical experimental limitations?

Going back to Eq. (1), we can rewrite this (using a convolution notation, rather than the integral form) as

$$
F(t)=g(t) \star R(t) \approx \delta(t)
$$

where $F(t)$ is the focal signal or source reconstruction, $R(t)$ is the recorded signal measured at the receiver location $B$ from the initial source propagation, and $g(t)$ is the signal necessary to be back propagated for focusing, also known as the result of the inverse filter, as will become evident. Here we restrict ourselves to looking only at signals between the two points $A$ and $B$ and thus have removed them from the notation. We also change from a Green function notation so as not to imply that we necessarily have an infinite bandwidth available. Further, since we only employ a single channel, we cannot utilize the singular value decomposition step proposed by Tanter et al. for noise rejection[5], and thus we rely on a high signal to noise ratio. From Eq. (2), we aim to approximate the focal signal $F(t)$ to a delta function by assuming that the recorded impulse response $R(t)$ contains information enough to produce an IF signal $g(t)$ to broadcast in our TR focusing procedure.

Deconvolution equates to inverse filtering by transforming to the frequency domain, thus Eq. (2) becomes

$$
F(\omega)=g(\omega) R(\omega) \approx 1
$$


which can easily be manipulated to provide $g(\omega)$ simply from measuring $R(\omega)$ (or rather from the FFT of $R(t))$. As such, we can now calculate the IF signal which should produce the most impulse-like TR focus from

$$
g(\omega)=\frac{1}{R(\omega)}=\frac{R^{*}(\omega)}{|R(\omega)|^{2}},
$$

where $*$ denotes a complex conjugate operation. This expression gives a mathematical expression for $g(\omega)$, but this may not be practical for experimental use in the event that there is limited bandwidth, significant background noise, or more precisely if $R(\omega)=0$ at any frequency. To avoid this we simply add a constant to the denominator to ensure that we never divide by 0 , hence we replace Eq. (4) by

$$
g(\omega)=\frac{R^{*}(\omega)}{|R(\omega)|^{2}+\epsilon},
$$

where $\epsilon$ is a constant related to the original received signal as

$$
\epsilon=\gamma \operatorname{mean}\left(|R(\omega)|^{2}\right) .
$$

The quantity $\gamma$, which is sometimes referred to as the waterlevel parameter [23], is an arbitrary constant chosen to optimally reduce the effect of noise introduced through the IF procedure. Here we use $\gamma=0.9$ for all experiments. The value 0.9 was chosen based on optimizing the focus energy in a process similar to that developed by Clayton et al.[23].

As the above derivation has been performed in the frequency domain, it is necessary to transform back to the time domain to recover the deconvolved signal, $g(t)$ to be used in the time reversal experiments. It is worth noting that this procedure produces a $g(t)$ that can be used directly for rebroadcasting, i.e., there is no need to perform a TR operation $g(t) \rightarrow g(-t)$ to obtain a focused signal. While this completes the deconvolution optimization procedure 
used in this paper, it is only a portion of the inverse filter procedure as defined by Tanter et al.[5], and utilized most recently by Gallot et al.[9]. For the full IF procedure a singular value decomposition (SVD) is often used for noise suppression, but since we are limited to a single channel in this study, SVD results in a simple normalization by a constant, and can thus be ignored. Therefore, the use of the deconvolution operation with a single channel requires a high signal to noise ratio.

From the above derivation it is easy to assume that the deconvolution is providing the best available signal for recreating a delta function. However, upon closer examination it is actually recreating a function related to the source function, which in previous studies of the inverse filter was designed, or assumed, to be impulse-like. If we separate the received signal into the Green function $(G(\omega))$ propagating the source function $(S(\omega))$ from $A$ to $B$, the transducer responses at $A$ and $B\left(T_{A}(\omega)\right.$ and $T_{B}^{\prime}(\omega)$ respectively, where prime and unprimed denote transmission and reception respectively) the received signal $R(\omega)$ can be represented as

$$
R=T_{B}^{\prime} G T_{A} S,
$$

where the frequency dependence $(\omega)$ of each function is implied. Deconvolving $R$ with a delta function in time, or applying the inverse filter in the frequency domain, and then propagating again from $A$ to $B$ we get a focal signal

$$
F=\frac{T_{B}^{\prime} G T_{A}}{T_{B}^{\prime} G T_{A} S}=\frac{1}{S} .
$$

Thus the IF sends back a frequency domain inverse of the forward information, while TR sends back a complex conjugate (or time reversal) of the forward information. The spectral division by $T_{B}^{\prime} G T_{A} S$ is unstable when this function has notches. This instability can be 
avoided, for example, by a water level regularization [23] where one does the following,

$$
\frac{1}{T_{B}^{\prime} G T_{A} S} \rightarrow \frac{\left(T_{B}^{\prime} G T_{A} S\right)^{*}}{\left|T_{B}^{\prime} G T_{A} S\right|^{2}+\epsilon} .
$$

Application of the inverse filter can thus also be used for source reconstruction for arbitrary sources (i.e., $S(t) \neq \delta(t)$, or $S(\omega) \neq 1$ ). To obtain the original source function it is only necessary to invert the focal signal $F(\omega)$ for the frequency domain representation, and inverse Fourier transform to obtain a source reconstruction as a function of time.

In the context of time reversal, the inverse filter is usually used for enhancing the temporal compression, assuming that the source signal was a delta function. By following the derivation above, another application would be to achieve an approximated delta function, limited by available bandwidth, from any source function. This application requires the source function to be known and multiplied, in the frequency domain, by the result of the inverse filter before broadcasting the signal for focusing. Using the regularization Eq. (9), the focal signal is then equal to

$$
F=\frac{S\left(T_{B}^{\prime} G T_{A} S\right)^{*}}{\left|T_{B}^{\prime} G T_{A} S\right|^{2}+\epsilon} T_{B}^{\prime} G T_{A} \approx 1 .
$$

In the absence of regularization $(\epsilon=0)$, the right hand side would be equal to 1 , which corresponds to a delta function $\delta(t)$ in the time domain. A nonzero regularization $(\epsilon>0)$ yields a temporal focus that differs from a true delta function. For bandlimited data, one can, at best, hope to retrieve a bandlimited delta function. By extension, to focus another arbitrary function $X$ from information acquired from the propagation of some other given source $S$, one can simply perform another multiplication such that the focal signal would be broadcasting the signal for focusing. Thus, using the regularization Eq. (9), the focal signal 
becomes

$$
F=\frac{X S\left(T_{B}^{\prime} G T_{A} S\right)^{*}}{\left|T_{B}^{\prime} G T_{A} S\right|^{2}+\epsilon} T_{B}^{\prime} G T_{A} \approx X
$$

Of course the arbitrary signal $X$ desired for focusing must have a spectral content that lies within the bandwidth of the original controlled, or known, source. Violating this requirement would reduce the fidelity of the reconstruction of $X$ in much the same way that the approximate delta function reconstruction is limited by the bandwidth available from the original source signal.

A final important point to be made here, before proceeding to the experimental validation, is the effect of transducer responses in the TR and IF procedures. From Eqs. (10) and (11) it is apparent that the the transducer responses do not completely cancel in the TR process, and thus there will be some added color to the resulting focal signal. The added color can be described as a ringing, or multiple converging wave fronts as recently shown by Anderson et al. [24]. However, this added coloring will be significantly reduced for the IF results as we will show in our experiments. Note that the transducer responses only cancel for reciprocal TR (forward from $A$ to $B$ and then backward from $A$ to $B$ ), whereas they don't necessarily cancel for standard TR (forward from $A$ to $B$ and then backward from $B$ to $A$ ) because the transducer responses in transmit and receive are not equal in general. Starting with Eq. (7) and following through the reciprocal TR process we can see that the focal signal becomes

$$
F=\left(T_{B}^{\prime} G T_{A} S\right)^{*} T_{B}^{\prime} G T_{A}=\left|T_{B}^{\prime}\right|^{2}\left|T_{A}\right|^{2} S^{*}
$$

with the ${ }^{*}$ denoting the complex conjugate. Thus in order to get the source reconstruction, one must know the transducer responses at both locations $A$ and $B$, or use transducers possessing flat frequency responses over the bandwidth of interest, as they each color the focal signal by the square of their frequency responses. 
Anderson, Wave Motion

\section{Experimental Validation: Temporal Characteristics}

The purpose of this study is to optimize focusing in experimental situations where only a single channel is used with reverberant signals in a closed cavity. With this in mind, it is prudent to test the methodology experimentally, as presented above. To do this, we used a sample made of fused quartz, $10 \times 10 \times 10 \mathrm{~cm}^{3}$. A source transducer $(1.27 \mathrm{~cm}$ diameter, 2 mm thick piezoelectric disk) was attached to the sample using epoxy. This defines, and fixes, our source position $A$. The receiver, a non-contact laser vibrometer (PSV 301, OFV 5000 controller, Polytec Inc.), was positioned at a point $B$ on the surface. As we cannot perform a standard TR experiment with a laser vibrometer, since it cannot act as both receiver and source, we performed reciprocal TR experiments, i.e., where the original source and back propagation signals are emitted from the same location/transducer, thus focusing the wave energy to the original, user-selected receiver location. This use of TR in a reciprocal sense is now a widely used form of TR, and indeed is the method originally used by Parvelescu[1] in the first known demonstration of TR in acoustics. It is also the method described analytically in detail in Sec. 2.

The source function, a $5 \mu$ shaped toneburst of $200 \mathrm{kHz}$ (shaped with a $\sin ^{2}$ envelope), was broadcast from the source transducer using a 12-bit arbitrary waveform generator with a conversion rate of $10 \mathrm{MHz}$. The response to this impulse was recorded by the laser vibrometer $(5 \mathrm{~mm} / \mathrm{s} / \mathrm{V}, 250 \mathrm{kHz}$ bandwidth) using a 14-bit digitizer also sampling at $10 \mathrm{MHz}$. The generator and digitizer were synced such that the source impulse was centered in a 3.2768 ms window, thus the first portion of the received signal $R(t)$ contains no causal signal. This also means that the TR focus will occur at $1.6384 \mathrm{~ms}$ during the rebroadcast. All excitations, i.e., for source TR and IF broadcasts, are performed at a maximum $100 \mathrm{Vpp}$.

Before we directly compare the temporal compression and focus abilities of both TR and the IF, we must first decide what $R(t)$ and $g(t)$ signals to use to create the focusing. In Fig. 1 
we illustrate four different IF methods of creating a focus of energy. For each of these four methods, we attempt to create a delta function and thus utilize the formulation in Eq. (10). During the forward propagation we record the incident vibration with the laser vibrometer at a location on the surface of the block, which arrives in the latter half of the $3.2768 \mathrm{~ms}$ window (see Fig. 1(a-1)). In reciprocal TR, we simply reverse this signal and broadcast it (see Fig. 1(a-2) and (a-3)) into the sample with the piezoelectric transducer to achieve focusing at the laser vibrometer location (see Fig. 1(a-4)). Before broadcasting the signal in reciprocal TR (and in other deconvolution methods) we can amplify the signal to maximize the focusing amplitude. In what we term the full deconvolution process, the deconvolution operation is performed on the full signal recorded during the forward propagation (see Fig. 1(b-1)) to obtain the signal (see Fig. 1(b-2) and (b-3)) used to create the focusing (see Fig. 1(b-4)). Another way to perform the deconvolution method is to take the full deconvolution result (see Fig. 1(b-2) and (c-2)) and set the second half of the signal $(t>1.6384 \mathrm{~ms})$ equal to zero (see Fig. 1(c-3)) and observe the focusing (see Fig. 1(c-4)). Finally, if we shorten the acquisition window to only the second half (1.6384 ms $<t \leq 3.2768 \mathrm{~ms}$ ), thus avoid any acausal portion of the signal, and use this signal (see Fig. 1(d-1)) in the deconvolution operation (see Fig. 1(d-2)), we can then zero pad this signal (see Fig. 1(d-3), the zero padding is done to ensure that the focal signals in each of the 4 processes are of the same length) before sending it back into the block to observe the focusing (see Fig. 1(d-4)).

In summary, Fig. 1 row (a) illustrates the signals used in the reciprocal TR process (blue colored signals), row (b) illustrates the signals used in the full deconvolution process (red colored signals), row (c) illustrates the signals used in the zeroed-acausal-portion deconvolution process (purple colored signals), and row (d) illustrates the signals used in the no-acausalportion signal deconvolution process (green colored signals). Fig. 1 column 1 depicts the signals obtained during the forward propagation step $(R(t))$, column 2 depicts the signals obtained from the deconvolution process $(g(t))$ performed on the signals in column 1, column 
3 depicts the signals that we send back into the block after allowing for some processing of the signals in column 2 (such as zeroing out portions of the signal or zero padding them), and column 4 depicts the focal signals from each process $(F(t))$. Note the reduction in amplitude, by a factor of about 3, that results from the deconvolution processes in comparison to the reciprocal TR process.

The focal signals recorded at $B$ due to the broadcast of each of the signals shown in Fig. 1, column (c), are now compared in Fig. 2. For clarity the colors used in Figs. 1 and 2 are consistent. The first noteworthy difference lies in the comparison of the original source signal with the focused signals resulting from reciprocal TR and the full deconvolution, Fig. 2(a). Here we see what has been reported in prior IF studies, i.e., that the IF produces a more temporally compressed focal signal. This is most easily seen by normalizing each signal to the total signal magnitude and calculating the fraction of the amount of energy in the original source $(1.6359<\tau<1.6409 \mathrm{~ms})$. Doing so we find that the original source contained $100 \%$ of its energy in that window (by definition), while the TR and IF reconstructions contain $14 \%$ and $78 \%$ respectively. This can also be seen in the envelopes shown in Fig. 2(c) over the full signal duration. While this type of quantification is new, this observation has been made previously[21].

In comparing the various deconvolution results, i.e., full, zeroed-acausal-portion, and noacausal-portion deconvolution processes, we see, Fig. 2(b), that in the focal interval shown, each method produces an identical result. However, in looking at the entire signal duration, we can see that not employing the acausal signal (either by zeroing it out before back propagation, Fig. 1 row (c), or by not including the signal recorded before the source emission, Fig. 1 row (d)) there are undesirable artifacts introduced at times away from the focus (raising the noise floor) decreasing the reconstruction percentage from $78 \%$ down to $54 \%$ and $63 \%$ respectively, indicating that the acausal portions of the signal are highly desirable to be used for any application where high signal fidelity is important or where multiple impulses may Page 13 
be focused successively with little separation, e.g., communications or lithotripsy. Another method used to quantify the improved temporal focus is through signal to noise ratio. We quantify the apparent signal to noise ratio by the ratio of the main maximum and the second largest maximum in the signal. This ratio measures how well the main maximum stands out above the rest of the signal. For full deconvolution, the ratio is 14.8 (Fig. 1 (b)-4). However, the ratio drops down to 10.3 for the result shown in (Fig. 1 (c)-4) where the acausal signal was zeroed out before emission. The ratio was 10.2 for the result shown in (Fig. 1 (d)-4) where the signal recorded before the source emission was not included. Hence the best method to use is the full deconvolution that benefits from a pseudo zero padding (the silence recorded before the source emission).

Before leaving the temporal domain and investigating the effect of the IF on the spatial focusing, we will look at source reconstruction as discussed in Sec. 2, Eqs. (10) and (11). To do so we alter our original source function, which we now call $S_{1}$ and is shown in Fig. 3(a), by applying a phase shift of $90^{\circ}$. We refer to this phase shifted source as $S_{2}$ shown in Fig. 3(b). As the only difference between the two sources is a phase shift, the two sources have equally defined bandwidths, thus the IF filter will apply equally well, or poorly, to the received signals from both. Relevant to our previous results on the implementation of the IF, the reconstruction procedures will employ the full deconvolution, as that has been demonstrated, above, to be the cleanest implementation. Figure 3 displays the results of the focusing procedures, i.e., source reconstruction (Eq. 11, with $X=1$ ) and limited band delta function (Eq. 10), compared to the two original source functions. A comparison of Fig. 3(a) and (b) shows that employing Eq. 11 (with $X=1$ ) produces a very good approximation of the original source functions. When multiplying by the source function prior to back-propagation, we can verify that the original phase information of the source is lost and the focal signal approximates a delta function to the extent possible with the available bandwidth. The fact that a true single point delta function is not achieved is of no 
surprise, as finite signal length and available bandwidth make this effectively impossible by any means.

In conclusion, the initial main objective for the use of deconvolution has been to improve the temporal focusing. We showed experimentally that simple deconvolution can achieve this. Beyond improving temporal compression of the focal signal, we also showed that the details of implementation of the IF is important, with the most robust being what we refer to as a full deconvolution. Finally, we showed the ability of the IF to be used for arbitrary source reconstruction or delta function approximation. All of the above demonstrations were limited to time domain observations. In the next section, we present one explanation, and supporting observations, for the improvement of spatial focusing with improved temporal compression.

\section{Improved temporal focusing leads to improved spatial}

\section{focusing}

In this section, we show that better temporal focusing implies better spatial focusing for the spherical average of the focus. We begin by first considering the wave field near its focal spot at $r=0$ and consider the medium to be locally homogeneous in that region. The solution of the Helmholtz equation in an acoustic, homogeneous medium can be written as

$$
p(r, \theta, \varphi, \omega)=\sum_{l=0}^{\infty} \sum_{m=-l}^{m=l} a_{l m} j_{l}(k r) Y_{l m}(\theta, \varphi),
$$

see Table 8.2 of Ref. [22]. In this expression $j_{l}$ denotes the spherical Bessel function, $Y_{l m}$ the spherical harmonics, and $k=\omega / c$, where $c$ is the wave speed. According to expressions (11.144) and (11.148) of Ref. [22], $j_{l}(0)=0$ for $l \geq 1$ and $j_{0}(0)=1$. This allows us to investigate the focus of the wave field by looking at the spherically symmetric component 
$l=0$. Using $Y_{00}=1 / \sqrt{4 \pi}$ (Table 12.3 of Ref. [22]), this means that at the focal point

$$
p(r=0, \theta, \varphi, \omega)=\frac{a_{00}}{\sqrt{4 \pi}}
$$

The properties of the wave field at the focal point thus only depend on the coefficient $a_{00}$. Since the $l=m=0$ component of the spherical harmonics expansion gives the spherically symmetric component of the wave field, the properties of the wave field at the focal point can only bear a relation to the spherically symmetric component of the wave field $(l=m=0)$. This implies that the properties of temporal focusing, defined as the time dependence of the wave field at $r=0$, can only be related to the spherically symmetric component of the spatial focusing.

In the following, we only analyze the spherically symmetric component $p_{l=0}(r, t)$ of the focus, because according to Fig. 4 (a) and (b), the central focal spot is nearly spherically symmetric. Using that $j_{0}(k r)=\sin (k r) / k r$, the spherically symmetric component of Eq. (13) is given by

$$
p_{l=0}(r, \omega)=p_{0} \frac{e^{-i k r}-e^{i k r}}{r}
$$

with $p_{0}=-a_{00} /(2 i k \sqrt{4 \pi})$. The coefficient $p_{0}$ depends on frequency. Using the Fourier convention $f(t)=\int p_{0}(\omega) e^{-i \omega t} d \omega$, and using that $k=\omega / c$, Eq. (15) corresponds, in the time domain, to

$$
p_{l=0}(r, t)=\frac{f(t+r / c)-f(t-r / c)}{r} .
$$

In this equation, $f(t+r / c)$ denotes the wave that is incident on the focus, and $f(t-r / c)$ the outgoing wave once it has passed through the focus. The field at the focus follows by Taylor expanding $f(t \pm r / c)$ in $r / c$ and taking the limit $r \rightarrow 0$, this gives

$$
p_{l=0}(r=0, t) \approx \frac{2}{c} f^{\prime}(t)
$$


In this expression and the following, each prime symbol denotes a time derivative. Equation (17) states that the wave field at the focus is the time derivative of the incoming wave field. Equation (17) gives the temporal properties of the focus. In order to get the spatial properties, we consider the wave field near the focal point at time $t=0$. Setting $t=0$ in Eq. (16), and making a third order Taylor expansion of $f( \pm r / c)$ gives

$$
p_{l=0}(r, t=0) \approx \frac{2}{c} f^{\prime}(0)+\frac{r^{2}}{3 c^{3}} f^{\prime \prime \prime}(0)
$$

Using Eq. (17) to eliminate $f$, gives

$$
p_{l=0}(r, t=0) \approx p_{l=0}(r=0, t=0)+\frac{1}{6 c^{2}} p_{l=0}^{\prime \prime}(r=0, t=0) r^{2} .
$$

This is a parabolic approximation for the wave field near the focus, with coefficients related to the wave field and the focus and its second time derivative. The radius $R$ of the focal spot can be estimated by setting the left hand side of Eq. (19) equal to zero, this gives

$$
R \approx \sqrt{6 c^{2}} \sqrt{\frac{-p_{l=0}(r=0, t=0)}{p_{l=0}^{\prime \prime}(r=0, t=0)}} .
$$

A good temporal focusing means that the temporal curvature of the wave field at the focal point is strong, this means that $-p^{\prime \prime} / p$ is large, and that the radius $R$ is small. Good temporal focusing thus implies that the radius in the spherically symmetric part of the spatial focus is small.

This claim of improved spatial focusing accompanying improved temporal compression can be experimentally observed by measuring the wave fields produced in the two focusing procedures, TR vs. IF. The surface of the fused quartz block was scanned with a laser vibrometer while each type of focus was produced. The improvement in the temporal focal quality of the IF procedure over TR has already been shown in Figures 1 and 2 . Figure 4 
displays the comparisons of the spatial extent of the two focusing procedures obtained with the scanning laser. The cross sectional displays of the data in Figures 4(c) and (d) show that the IF focus is $17 \%$ narrower as measured across the full width at half of the respective maxima (i.e., $2-3 \mathrm{~mm}$ over $\sim 1 \mathrm{~cm}$ ).

While the spatial extent of the focus appears to be smaller using the IF method, confirming the theory of improved temporal compression resulting in improved spatial focusing, two other features are also noticeable. First, in no particular order, is the larger degree of uniformity $(\approx 0)$ of the IF wave field away from the focus, while the TR focus is much more structured across the imaged region. Second is the fact that the maximum achieved amplitude using the IF is only half that of the TR method, despite the fact each signal was normalized and rescaled to the same maximum voltage (100 Vpp) prior to rebroadcast.

\section{Conclusion}

Here we have explored a simple method for determining the optimal signal to be used in a TR experiment in order to maximally compress the focus as an approximate delta function (with limited bandwidth) in both time and space or for optimal signal reconstruction. Creation of an approximate delta function technically requires knowledge of the source signal, whereas source reconstruction requires a second inverse filter operation in post processing. Previous implementations of the inverse filter operation avoided the need to know the source signal by utilizing impulse like source signals. This method has been experimentally verified, albeit without fully varying all possible parameters. A more detailed study is currently underway to quantify the effects due to variations in available bandwidth, and material properties

(e.g., homogeneous vs. heterogeneous media, range of attenuations, wave speeds, length of scattered signal used, etc.)

While temporal and spatial focusing have been enhanced with this method, there is a cost Page 18 
in the maximum achieved amplitude by a factor of 2 or 3. This fact, coupled with the effect of the acausal portion of $g(t)$ may lead one to think simplistically about $g(t)$ as containing a signal to focus the energy at the appropriate time and place, and another signal that, simultaneously, actively cancels noise at all other times and locations. Thus the IF focus is cleaner and more impulse-like, but not as strong, as some of the energy being transmitted is exerted in suppressing sidelobes, spatially and temporally.

We have also shown, theoretically and experimentally, that if one has good temporal focusing, the radius in the spherically symmetric part of the spatial focus is small. This concept can turn out to be useful in future research because it allows one to work on improving the temporal focusing in order to improve the spatial focusing, e.g., communicating to a precise location or focusing high intensity ultrasound for lithotripsy without damaging the surrounding area, to name a few. When the focal spot is far from being spherically symmetric, as may be the case where the receiver aperture is small or the duration of the recording of scattered waves is short, an improved temporal focus does not necessarily imply an improved spatial focus. 


\section{${ }_{35}$ List of Figures}

1 Time reversed signals used for back propagation, to create a delta function (Eq. 10), (a) (blue): original response $R(-t)$ due to a $5 \mu \mathrm{s} 200 \mathrm{kHz}$ tone burst; (b) (red): full signal $g(t)$ from the deconvolution procedure described herein; (c) $g(t)$ as obtained from utilizing only the causal portion of $R(t)$ and zero padding for the acausal portion; (d) $g(t)$ from (b) with the acausal portion (i.e., $t>1.6384 \mathrm{~ms}$ ) set equal to $0 . \ldots \ldots \ldots \ldots$

2 Normalized focal signals resulting from the back propagation of the TR signals shown in Fig. 1, compared with the original source function. (a) \& (b) illustrate the structure of the signals near the focal time, each of which have been normalized to the total signal magnitude to accurately compare the temporal compression of the signal. (c) \& (d) are envelopes of the focused signals for the entire duration of the signal. Envelopes are presented normalized to the maximum peak amplitude of the velocity to illustrate the difference in perceived signal to noise (i.e., the signal amplitude at the focal time compared to all other times in the signal.) . . . . . . . . . . . . . . . . 
3 Source reconstruction and "delta" function results using deconvolutions. The original source is shown in comparison to the source reconstruction (i.e., from Eq. 11, with $X=1$ ) and the delta function approximation (i.e., from Eq. 10). (a) Comparison using $S_{1}$ a $5 \mu s 200 \mathrm{kHz}$ toneburst with a maximum at 1.6384 ms. (b) Comparison using a source function $S_{2}$ of identical bandwidth to $S_{1}$ but with a $90^{\circ}$ phase shift. . . . . . . . . . . . . . .

4 (a) two dimensional image of the wave field at the time of focus using classical TR. (b) two dimensional image of the wave field at the time of focus using the IF. (c) one dimensional line-outs from (a) and (b) at $y=25 \mathrm{~mm}$. (d) one dimensional line-outs from (a) and (b) at $x=25 \mathrm{~mm}$. Note that the $\mathrm{x}$ and $\mathrm{y}$ axes of (c) and (d) have been truncated to show the narrowing of the IF foci compared to the TR foci. . . . . . . . . . . . . . . . . . 25

5 Brian Anderson . . . . . . . . . . . . . . . . . . . . . 28

6 Johannes Douma . . . . . . . . . . . . . . . . . 28

7 TJ Ulrich . . . . . . . . . . . . . . . . . . . . . 29

$8 \quad$ Roel Snieder . . . . . . . . . . . . . . . . . . . . . 29 
Anderson, Wave Motion
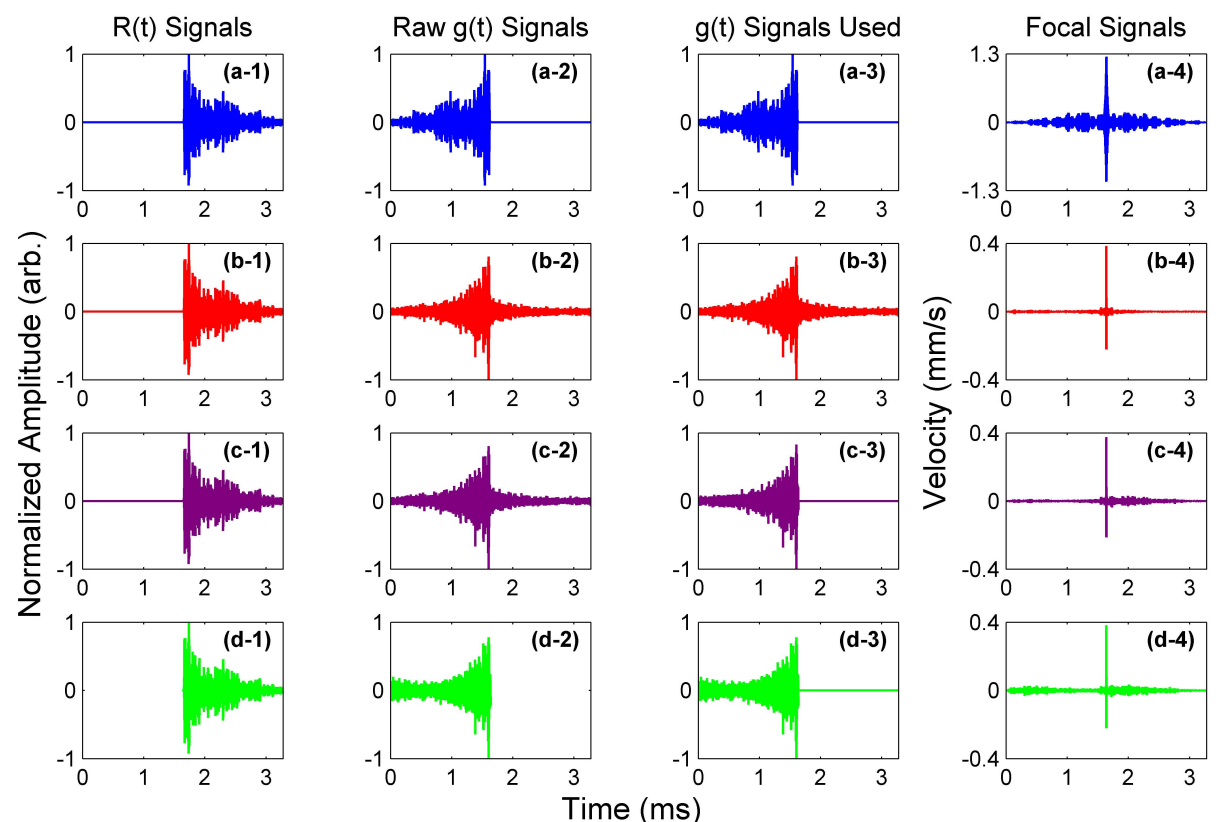

Figure 1: Time reversed signals used for back propagation, to create a delta function (Eq. 10), (a) (blue): original response $R(-t)$ due to a $5 \mu \mathrm{s} 200 \mathrm{kHz}$ tone burst; (b) (red): full signal $g(t)$ from the deconvolution procedure described herein; (c) $g(t)$ as obtained from utilizing only the causal portion of $R(t)$ and zero padding for the acausal portion; (d) $g(t)$ from (b) with the acausal portion (i.e., $t>1.6384 \mathrm{~ms}$ ) set equal to 0. 
Anderson, Wave Motion
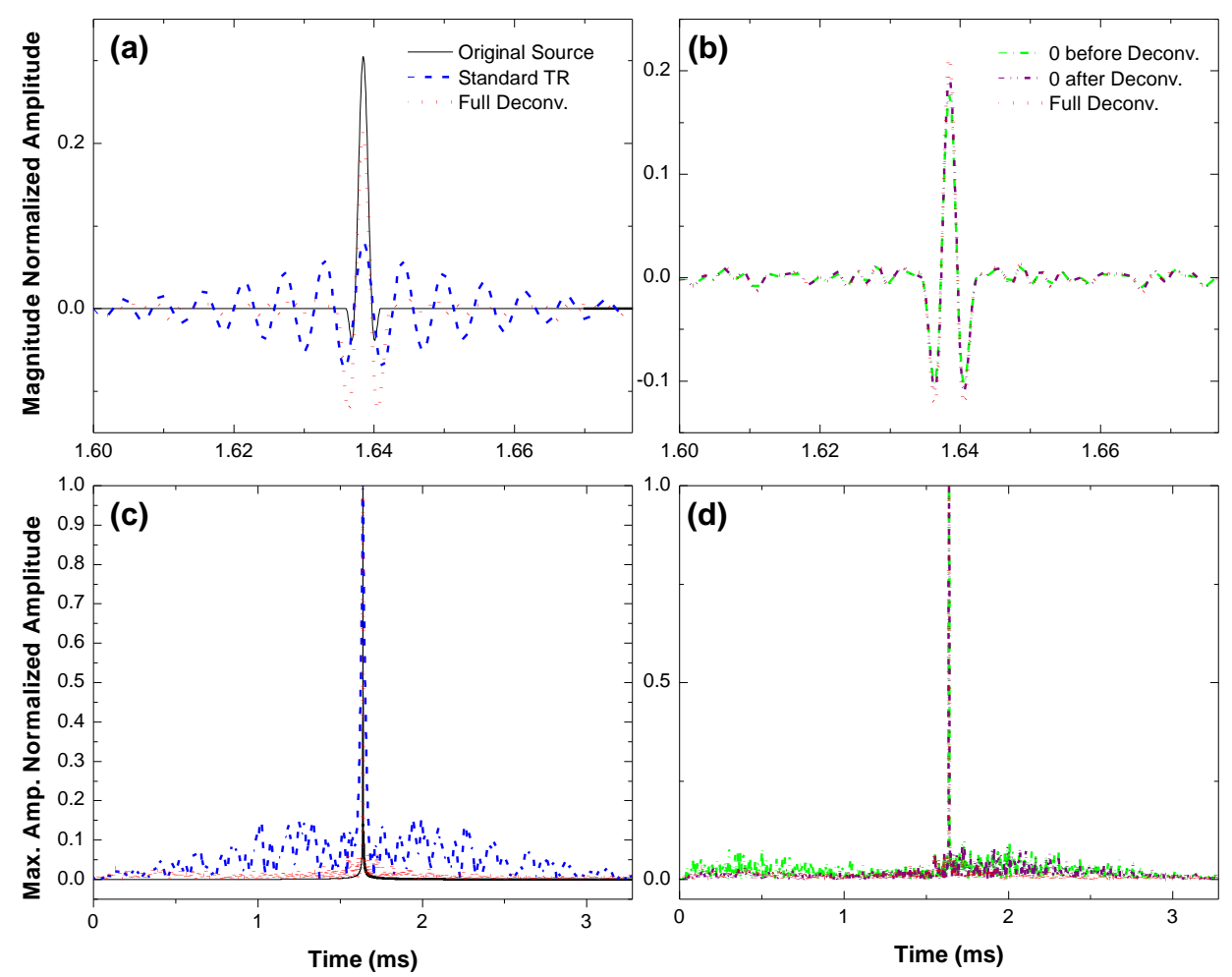

Figure 2: Normalized focal signals resulting from the back propagation of the TR signals shown in Fig. 1, compared with the original source function. (a) \& (b) illustrate the structure of the signals near the focal time, each of which have been normalized to the total signal magnitude to accurately compare the temporal compression of the signal. (c) \& (d) are envelopes of the focused signals for the entire duration of the signal. Envelopes are presented normalized to the maximum peak amplitude of the velocity to illustrate the difference in perceived signal to noise (i.e., the signal amplitude at the focal time compared to all other times in the signal.) 
Anderson, Wave Motion

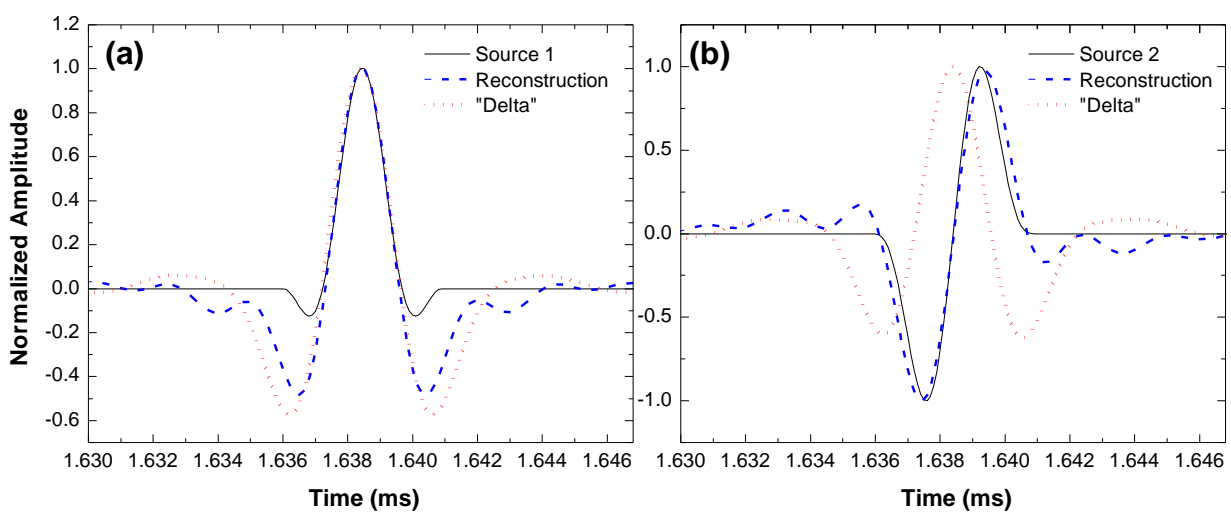

Figure 3: Source reconstruction and "delta" function results using deconvolutions. The original source is shown in comparison to the source reconstruction (i.e., from Eq. 11, with $X=1$ ) and the delta function approximation (i.e., from Eq. 10). (a) Comparison using $S_{1}$ a $5 \mu s 200 \mathrm{kHz}$ toneburst with a maximum at $1.6384 \mathrm{~ms}$. (b) Comparison using a source function $S_{2}$ of identical bandwidth to $S_{1}$ but with a $90^{\circ}$ phase shift. 
(a)
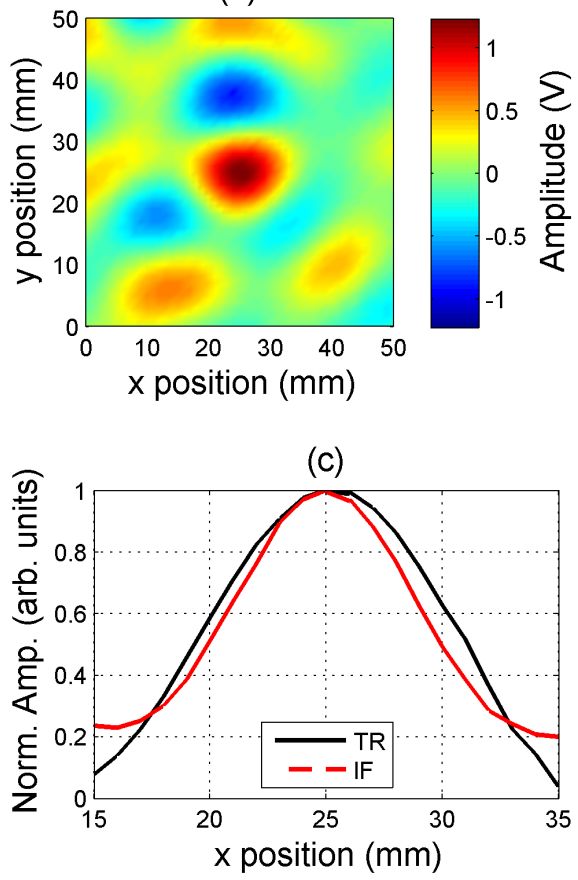

(b)

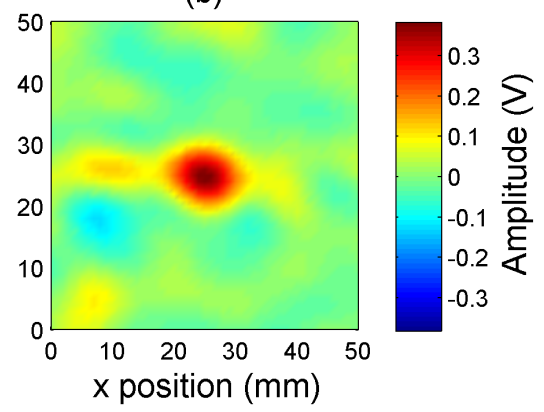

(d)

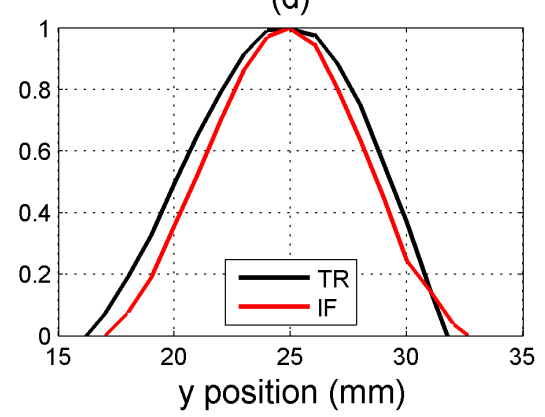

Figure 4: (a) two dimensional image of the wave field at the time of focus using classical TR. (b) two dimensional image of the wave field at the time of focus using the IF. (c) one dimensional line-outs from (a) and (b) at $y=25 \mathrm{~mm}$. (d) one dimensional line-outs from (a) and (b) at $x=25 \mathrm{~mm}$. Note that the $\mathrm{x}$ and $\mathrm{y}$ axes of (c) and (d) have been truncated to show the narrowing of the IF foci compared to the TR foci. 
Anderson, Wave Motion

\section{${ }_{427}$ Acknowledgements}

We would like to thank the anonymous reviewers for their critical and constructive commentive. Work supported by Los Alamos National Laboratory Institutional Support (LDRD).

\section{Vitae}

Brian Anderson is a staff scientist in the Geophysics Group of the Los Alamos National Laboratory, where he also completed a postdoc before full time employment. Prior to working at Los Alamos he was a visiting assistant professor of acoustics in the Physics and Astronomy Dept. at Brigham Young University (BYU) for 3 years. He earned his Ph.D. in Acoustics from The Pennsylvania State University in 2006. He earned his M.S. and B.S. degrees in Physics from BYU in 2003 and 2001 respectively. He currently serves as an Associate Editor for the Journal of the Acoustical Society of America.

Johannes recently graduated from Colorado school of mines with a master of science. He was part of the center for wave phenomena with Dr. Roel Snieder as his advisor. He completed a project to optimize time reversal focusing through deconvolution for acoustic waves with Prof. Snieder and Los Alamos National Laboratory researchers TJ Ulrich and Brian E. Anderson. Additionally, he recently worked together with visiting researcher Dr. Ernst Niederleithinger on applying his method to a concrete block with embedded receivers at the civil engineering lab in Mines. He then began applying the method towards improving the locating of microseismic events. This work has been completed for both the acoustic case as well as the elastic case.

Timothy James (TJ) Ulrich II obtained his Ph.D. in Physics at the University of Nevada Reno (UNR) after an M.S. degree in Physics and a B.S. in Materials Science and Engineering, also at UNR. Ulrich came to LANL in 2005 as a post-doc, later becoming a staff 
member (R\&D Scientist) in 2008. His work includes developing new acoustics-based experimental tools and techniques for materials characterization and nondestructive evaluation as well as developing instrumentation, software and performing experiments in support of the laboratory mission as well as for industrial applications.

Roel Snieder holds the Keck Foundation Endowed Chair of Basic Exploration Science at the Colorado School of Mines. He received in 1984 a Masters degree in Geophysical Fluid Dynamics from Princeton University, and in 1987 a Ph.D. in seismology from Utrecht University. In 1993 he was appointed as professor of seismology at Utrecht University, where from 1997-2000 he was appointed as Dean of the Faculty of Earth Sciences. 
Anderson, Wave Motion

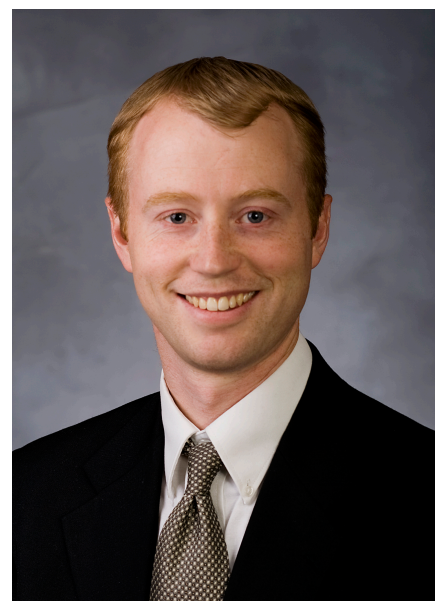

Figure 5: Brian Anderson

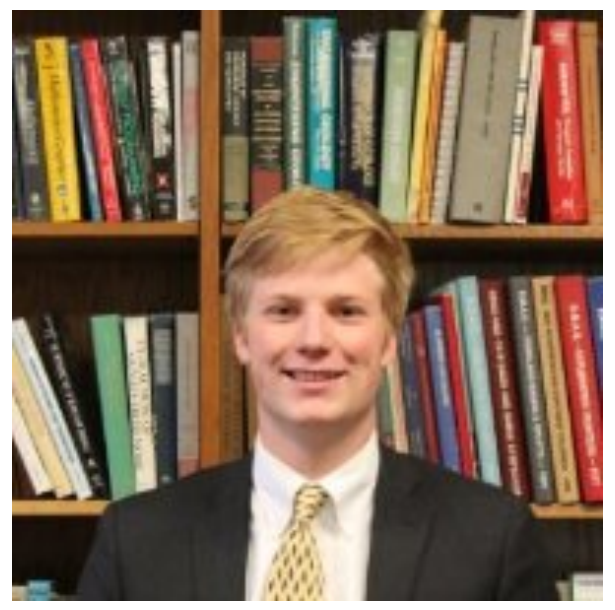

Figure 6: Johannes Douma

Page 28 
Anderson, Wave Motion

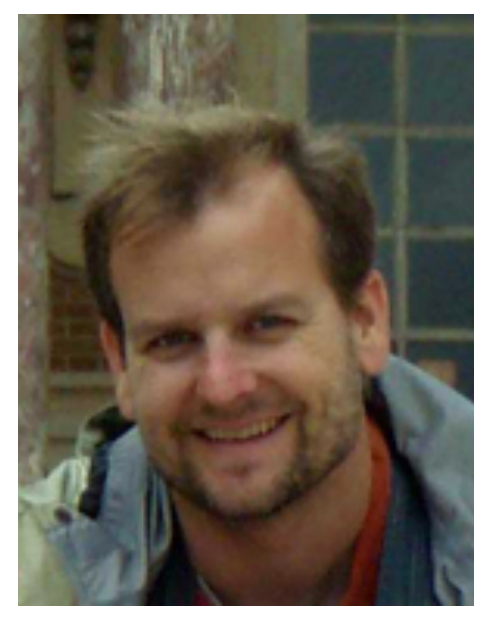

Figure 7: TJ Ulrich

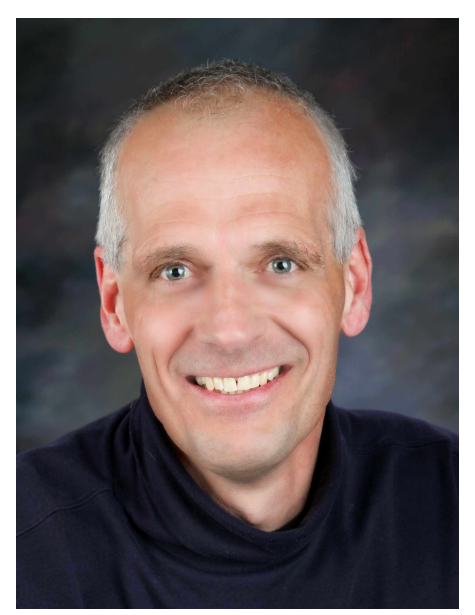

Figure 8: Roel Snieder 


\section{« Bibliography}

[1] A. Parvulescu and C. Clay. "Reproducibility of signal transmission in the ocean." Radio Elec. Eng., 29:223-228, 1965.

[2] M. Fink. "Time reversed acoustics." Physics Today, 50(3):34-40, 1997.

[3] B.E. Anderson, M. Griffa, C. Larmat, T.J. Ulrich, and P.A. Johnson. "Time reversal." Acoustics Today, 4(1):5-16, 2008.

[4] C.S. Larmat, R.A. Guyer, and P.A. Johnson. "Time-reversal methods in geophysics." Physics Today, 63(8):31-35, 2010.

[5] M. Tanter, J.-L. Thomas, and M. Fink. "Time reversal and the inverse filter." J. Acoust. Soc. Am., 108:223-234, 2000.

[6] M. Tanter, J.-F. Aubry, J. Gerber, J.-L. Thomas, and M. Fink. "Optimal focusing by spatio-temporal filter. I. Basic principles." J. Acoust. Soc. Am., 110:37-47, 2001.

[7] G. Montaldo, M. Tanter, and M. Fink. " Real time inverse filter focusing through iterative time reversal." J. Acoust. Soc. Am., 115:768-775, 2004.

[8] F. Vignon, J.-F. Aubry, A. Saez, M. Tanter, D. Cassereau, G. Montaldo, and M. Fink. "The Stokes relations linking time reversal and the inverse filter." J. Acoust. Soc. Am., 119:1335-1346, 2006. 
[9] T. Gallot, S. Catheline, P. Roux, and M. Campillo. “A passive inverse filter for Green's function retrieval." J. Acoust. Soc. Am., 131:EL21-EL27, 2011.

[10] F. Ciampa and M. Meo. "Acoustic emission localization in complex dissipative anisotropic structures using a one channel reciprocal time reversal method." J. Acoust. Soc. Am., 130(1):168-175, 2011.

[11] M. Meo. and F. Ciampa "Impact detection in anisotropic materials using a time reversal approach." Struct. Health Monitor., 11(1):43-49, 2012.

[12] V. Bertaix, J. Garson, N. Quieffin, S. Catheline, J. Derosny, and M. Fink. " Timereversal breaking of acoustic waves in a cavity." Am. J. of Phys., 72(10):1308, 2004.

[13] P. Roux and M. Fink. "Time reversal in a waveguide: study of the temporal and spatial focusing". J. Acoust. Soc. Am., 107(5 Pt 1):2418-2429, May 2000.

[14] J.-F. Aubry, M. Tanter, J. Gerber, J.-L. Thomas, and M. Fink. "Optimal focusing by spatio-temporal filter. II. Experiments. Application to focusing throuh absorbing and reverberating media." J. Acoust. Soc. Am., 110:48-58, 2001.

[15] B. L. G. Jonsson, M. Gustafsson, V. H. Weston, and M. V. de Hoop. "Retrofocusing of acoustic wave fields by iterated time reversal." SIAM J. Appl. Math., 64(6):1954-1986, 2004.

[16] R. Daniels and R. Heath. "Improving on time reversal with MISO precoding." In Procedings of the Eighth International Symposium of Wireless Personal Communications Conference, Aalborg, Danmark, P.VI-124 - P.VI-129, 2005.

[17] R.C. Qiu, C. Zhou, N. Guo, and J.Q. Zhang. "Time reversal with MISO for ultrawideband communications: experimental results." IEEE Antennas and Wireless Propagation Lett., 5:1-5, 2006. 
[18] P. Blomgren, P. Kyritsi, A. Kim, and G. Papanicolaou. "Spatial focusing and intersymbol interference on multiple-input-single-output time reversal communication systems." IEEE J. Oceanic Eng., 33:341-355, 2008.

[19] C. Zhou, N. Guo, and R.C. Qiu. "Experimental results on multiple-input single-outpot (MISO) time reversal for UWB stystems in an office environment." In MILCOM'06 proceedings of the 2006 IEEE conference on military comunications, pages 1299-1304, Piscataway, NJ, 2006. IEEE Press.

[20] C. Zhou and R.C. Qiu. "Spatial focusing of time-reversed UWB electromagnetic waves in a hallway enviroment:." In Proceedings of the Thirty Eighth symposium on System Theory, pages 318 - 322, 2006.

[21] B. Van Damme, K. Van Den Abeele, Y. Li, and O. Bou Matar "Time reversed acoustics techniques for elastic imaging in reverberant and nonreverberant media: An experimental study of the chaotic cavity transducer concept." In J. Appl. Phys., 95:104910, 2011.

[22] G.B. Arfken and H.J. Weber. "Mathematical methods for physicists." Harcourt, Amsterdam, 4th edition, pages 477-739, 2001.

[23] R. Clayton and R. Wiggins. "Source shape estimation and deconvolution of teleseismic bodywaves." Geophys. J. Royal Astron. Soc., 47:151-177, 1976.

[24] B. E. Anderson, T. J. Ulrich, and P.-Y. Le Bas. "Comparison and visualization of the focusing wave fields of various time reversal techniques in elastic media." J. Acoust. Soc. Am., 134(6), EL527-EL533 (2013). 